\title{
Structure of liquid phase change material AgInSbTe from density functional/ molecular dynamics simulations
}

J. Akola, and R. O. Jones

Citation: Appl. Phys. Lett. 94, 251905 (2009);

View online: https://doi.org/10.1063/1.3157166

View Table of Contents: http://aip.scitation.org/toc/apl/94/25

Published by the American Institute of Physics

\section{Articles you may be interested in}

Nanoscale nuclei in phase change materials: Origin of different crystallization mechanisms of $\mathrm{Ge}_{2} \mathrm{Sb}_{2} \mathrm{Te}_{5}$ and AgInSbTe

Journal of Applied Physics 115, 063506 (2014); 10.1063/1.4865295

Nucleation of AgInSbTe films employed in phase-change media

Journal of Applied Physics 99, 064907 (2006); 10.1063/1.2184428

Crystallization kinetics of sputter-deposited amorphous AgInSbTe films Journal of Applied Physics 90, 3816 (2001); 10.1063/1.1405141

Evidence for thermally assisted threshold switching behavior in nanoscale phase-change memory cells Journal of Applied Physics 119, 025704 (2016); 10.1063/1.4938532

Investigation of switching region in superlattice phase change memories AIP Advances 6, 105104 (2016); 10.1063/1.4964729

Low power phase change memory switching of ultra-thin $\mathrm{In}_{3} \mathrm{Sb}_{1} \mathrm{Te}_{2}$ nanowires

Applied Physics Letters 109, 213103 (2016); 10.1063/1.4968510

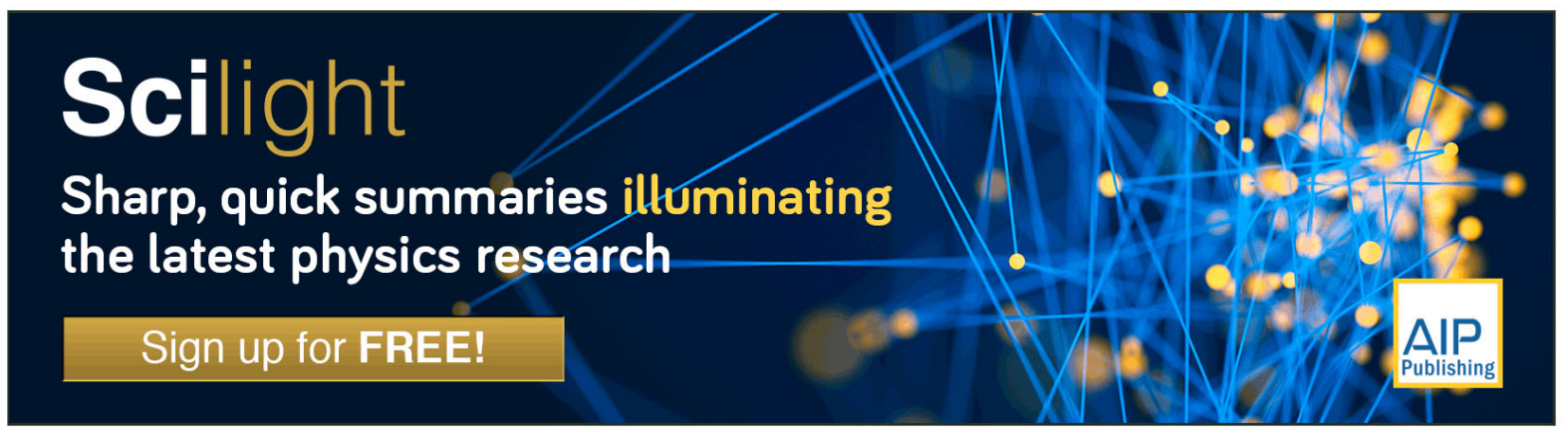




\title{
Structure of liquid phase change material AgInSbTe from density functional/molecular dynamics simulations
}

\author{
J. Akola ${ }^{1,2,3}$ and R. O. Jones ${ }^{1, a)}$ \\ ${ }^{1}$ Institut für Festkörperforschung, Forschungszentrum Jülich, D-52425 Jülich, Germany \\ ${ }^{2}$ Nanoscience Center, Department of Physics, P.O. Box 35, FI-40014 University of Jyväskylä, Finland \\ ${ }^{3}$ Department of Physics, Tampere University of Technology, P.O. Box 692, FI-33101 Tampere, Finland
}

(Received 20 April 2009; accepted 28 May 2009; published online 23 June 2009)

\begin{abstract}
The liquid phase of the AgInSbTe phase change material $\mathrm{Ag}_{3.5} \mathrm{In}_{3.8} \mathrm{Sb}_{75.0} \mathrm{Te}_{17.7}$ has been studied using molecular dynamics/density functional simulations. The calculated structure factor and total pair distribution function agree very well with high-energy x-ray diffraction measurements. We find that: (a) there are signs of medium-range order, (b) $\mathrm{Ag}$ and In prefer to be near Te rather than $\mathrm{Sb}$ atoms, and promote octahedral coordination in association with $\mathrm{Te}$, (c) $\mathrm{Ag}$ is the most mobile element, has the shortest bonds (2.8-2.9 $\AA$ ) and the highest coordination (5.5), and is anionic with the largest effective charge, and (d) there are few cavities (4\% of volume). () 2009 American Institute of Physics. [DOI: 10.1063/1.3157166]
\end{abstract}

Phase change materials involve the rapid and reversible change between nanoscale amorphous $(a-)$ and crystalline $(c-)$ spots in a polycrystalline film, and play central roles in rewritable media and nonvolatile computer memory. ${ }^{1}$ Two families of chalcogenide alloys dominate practical applications: (a) GeTe-Sb ${ }_{2} \mathrm{Te}_{3}$ (GST) pseudobinary alloys, ${ }^{2}$ and (b) AgInSbTe (AIST) alloys near the SbTe eutectic composition $\left(\mathrm{Sb}_{70} \mathrm{Te}_{30}\right){ }^{3}$ DVD-RAM and Blu-ray Disc are examples of the former, CD-RW and DVD-RW represent the latter. Understanding the phase change mechanisms is impossible without structural information, and there has been much speculation about the structure of the different alloy phases. Although these are complicated by the presence of three and four elements, respectively, significant progress has been made recently in both chalcogenide families.

A reverse Monte Carlo analysis of high-energy x-ray diffraction (HEXRD) data from the prototype GST material $a-\mathrm{Ge}_{2} \mathrm{Sb}_{2} \mathrm{Te}_{5}$ (Ref. 4) indicated that the structure was dominated by ring structures also found in the crystal. Extensive density functional (DF) calculations ${ }^{5-7}$ on liquid $(\ell-)$ and $a$-GST extended this picture and showed that mediumranged order exists among Te atoms, and that cavities and $A B A B$ squares $(A: \mathrm{Ge} / \mathrm{Sb}, B: \mathrm{Te})$ characterize these materials and their phase changes. Of particular interest is the timeresolved x-ray diffraction study of crystallization in $\mathrm{Ge}_{2} \mathrm{Sb}_{2} \mathrm{Te}_{5}$ and $\mathrm{Ag}_{3.5} \mathrm{In}_{3.8} \mathrm{Sb}_{75.0} \mathrm{Te}_{17.7}{ }^{8}$ The results are consistent with the different crystallization mechanisms: nucleation and subsequent growth in GST, growth of the surrounding crystalline edge in AIST. ${ }^{9}$ The amorphous marks in AIST films are more sharply defined along the crystal boundary, resulting in higher stability at elevated temperatures, lower jitter, ${ }^{9}$ and faster crystallization than in GST. ${ }^{8}$ Crystallization is the rate-limiting step in a write/erase cycle, and the addition of small amounts of $\mathrm{Ag}$ and $\mathrm{In}$ to $\mathrm{Sb} / \mathrm{Te}$ alloys increases both crystallization temperature ${ }^{10}$ and thermal stability. ${ }^{11}$

The liquid structures of phase change materials ${ }^{12}$ are crucial to amorphization and crystallization. The former results from quenching the liquid and the latter via an undercooled liquid state, but little is known about the structures

${ }^{a)}$ Electronic mail: r.jones@fz-juelich.de. involved. Here we perform DF calculations with molecular dynamics (MD) to study liquid $\mathrm{Ag}_{3.5} \mathrm{In}_{3.8} \mathrm{Sb}_{75.0} \mathrm{Te}_{17.7}$, which has been investigated previously below the melting point, ${ }^{8,13}$ and compare the results with HEXRD measurements. ${ }^{14}$ DF calculations are particularly valuable in AIST alloys, because the atomic radii (and bond lengths) and atomic numbers (4752) of the components are similar. X-ray scattering methods do not discriminate well between them, and the neutron scattering lengths of $\mathrm{Ag}, \mathrm{Sb}$, and Te differ by less than $10 \%{ }^{15}$

Combined MD/DF calculations ${ }^{5}$ were performed with the CPMD package, ${ }^{16}$ using the PBEsol approximation ${ }^{17}$ for the exchange-correlation energy. The initial structure contained 640 atoms ( $23 \mathrm{Ag}, 25 \mathrm{In}, 480 \mathrm{Sb}$, and $112 \mathrm{Te}$ ) distributed randomly in a cubic cell (size $27.087 \AA$ ) appropriate to the density of the liquid $\left(0.03220\right.$ atoms $\left./ \AA^{3}\right) .{ }^{18}$ The $4 d$ levels in $\mathrm{Ag}$ and In are relatively shallow and must be included in the "valence" configuration. Convergence of the calculation then requires a higher kinetic energy cutoff of the plane wave basis (60 Ry) than used in our work on GST alloys (20

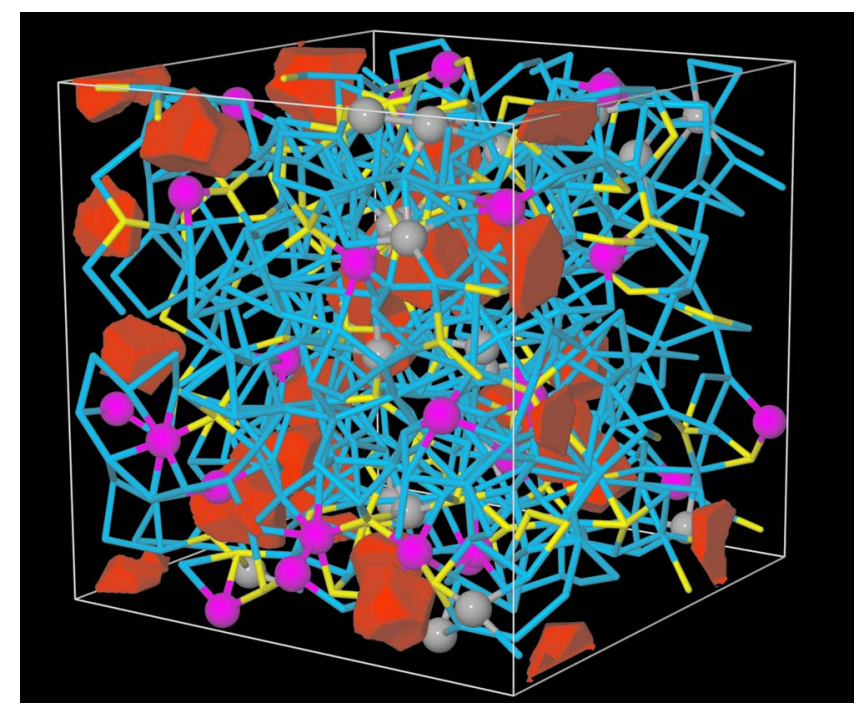

FIG. 1. (Color online) Snapshot of $\ell$-AIST (Ag: silver, In: magenta, Sb: blue, and Te: yellow). The dopants (Ag, In) are depicted with small spheres, the cavities are shown in red. 

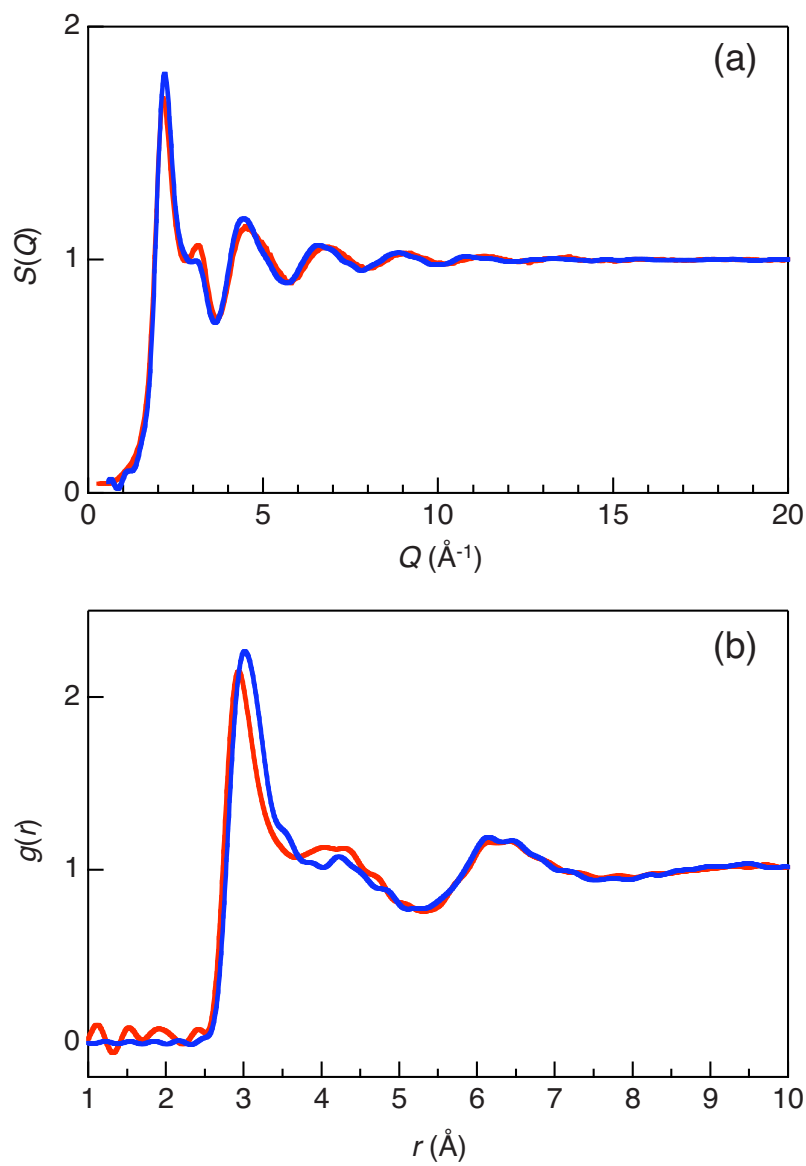

FIG. 2. (Color online) (a) Structure factor $S(Q)$ in $\ell$-AIST. (b) Total PDF $g(r)$ (XRD weights). Red (gray, oscillating at low $r$ ): XRD (862 K), using $Q_{\max }=25 \AA^{-1}$ in the Fourier transform, blue (black): present simulation $(850 \mathrm{~K})$.

Ry), ${ }^{5}$ and the computational demands are much greater. Further details are given in the supplementary information (SI) ${ }^{19}$ The simulation was initiated at $3000 \mathrm{~K}$, followed by cooling to $850 \mathrm{~K}\left(577^{\circ} \mathrm{C}, 30 \mathrm{ps}\right.$, and time step of $\left.4.8 \mathrm{fs}\right)$ and data collection $(20 \mathrm{ps}$ and time step of $3.0 \mathrm{fs})$. Figure 1 shows a snapshot of the structure with emphasis on small cavities (voids) and the sparsely distributed $\mathrm{Ag}$ and In atoms. On average there are only 22 cavities in the 640 atom sample, and their total volume $(\sim 4 \%)$ is much less than in $\ell$-GST $(\sim 14 \%)$ or $a$-GST $(\sim 12 \%)$, where they are crucial to the phase change mechanism. ${ }^{5}$

The calculated structure factor $S(Q)$ and the total pair distribution function (PDF) $g(r)$ were determined from 13 snapshots of structures taken at $850 \mathrm{~K}$. The agreement between theory and the XRD data is very good for $S(Q)$ [Fig. 2(a)] and $g(r)$ [Fig. 2(b)]. The minor deviations in $g(r)$ near the first peak (maxima at 2.94 and $3.02 \AA$, respectively) and

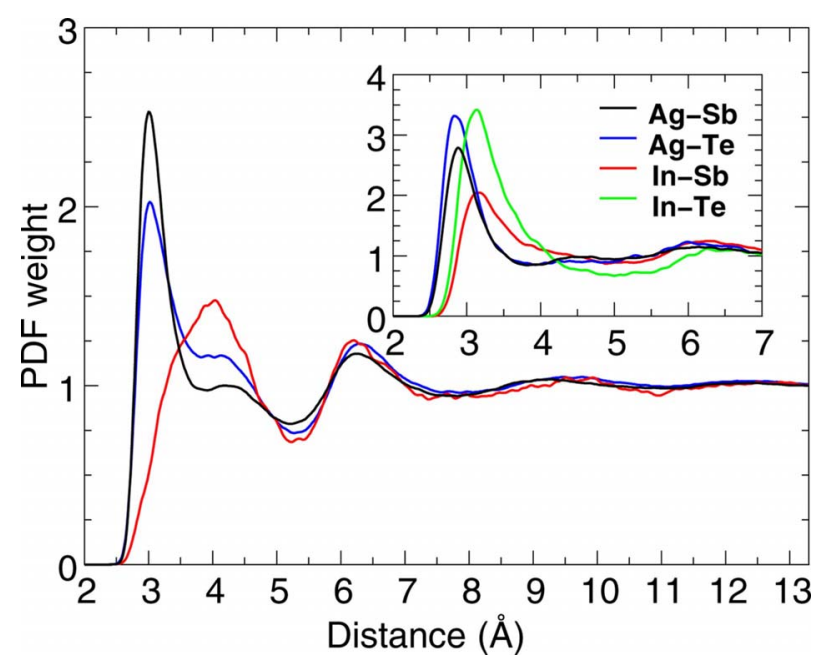

FIG. 3. (Color online) Partial PDF in AIST at $850 \mathrm{~K}$. Black: Sb-Sb, blue: $\mathrm{Sb}-\mathrm{Te}$, and red: $\mathrm{Te}-\mathrm{Te}$. Inset: Ag-Sb, Ag-Te, In-Sb, and In-Te.

the weak second maximum at $4.2 \AA$ suggest that the calculated coordination numbers may be slightly larger than the experimental estimates. Seven partial PDFs $g_{i j}(r)$ are shown in Fig. 3. Ag-Ag, $\mathrm{Ag}-\mathrm{In}$, and $\mathrm{In}-\mathrm{In} \mathrm{PDF}$ are given in the SI, ${ }^{19}$ where we also tabulate the dependence of coordination number on the choice of cutoff.

As in $\ell$-GST, ${ }^{5}$ there are signs of medium-range order and very few $\mathrm{Te}-\mathrm{Te}$ contacts. Bonds between $\mathrm{In}$ and $\mathrm{Sb} / \mathrm{Te}$ are longer (by $0.2-0.3 \AA$ ) than bonds between $\mathrm{Ag}$ and $\mathrm{Sb} / \mathrm{Te}$, and the first broad peak in the In PDF extends beyond $4 \AA$. Both minority (dopant) atoms Ag and In prefer Te neighbors over $\mathrm{Sb}$, a behavior also observed in EXAFS measurements of amorphous $\mathrm{Ag}_{5} \mathrm{In}_{5} \mathrm{Sb}_{60} \mathrm{Te}_{30} \cdot{ }^{10}$ However, the effect on total coordination is countered by the much higher $\mathrm{Sb}$ concentration. There are very few bonds among minority atoms $\mathrm{Ag}$ and $\mathrm{In}(\mathrm{Ag}-\mathrm{Ag}, \mathrm{Ag}-\mathrm{In}$, and $\mathrm{In}-\mathrm{In})$. The total coordination numbers (Table I) indicate that Ag and In increase the overall coordination, and they tend to form bond angles of $90^{\circ}$ when associated with Te. Te atoms also enhance the octahedral character around $\mathrm{Sb}$, including some nearly linear $\mathrm{Te}-\mathrm{Sb}-\mathrm{Te}$ configurations $\left(\lessgtr 180^{\circ}\right){ }^{19}$

DF calculations lead to electron density distributions and to energy eigenvalues that reflect the underlying band structure. The calculated density of states of the valence electrons (Fig. 4) shows $\mathrm{Sb} / \mathrm{Te} \sigma$-and $\pi$-bands separated by a gap at $-6.5 \mathrm{eV}$, and there are contributions from the minority atoms at -14.5 (In 4d) and $-4.5 \mathrm{eV}(\mathrm{Ag} 4 d$ and In 5s). The In $4 d$ orbital is close to the $\sigma$-band $(\mathrm{Sb} / \mathrm{Te})$ starting at $-13 \mathrm{eV}$, which justifies our assignment of the $4 d$ levels of $\mathrm{Ag}$ and In as valence electrons. There is neither a band gap nor a pronounced minimum at the Fermi energy, although band gaps

TABLE I. Coordination numbers $n_{\mathrm{X}}$ (cutoff 3.3-3.5 $\AA$, see SI), bond distances with $\mathrm{Sb}\left(r_{\mathrm{X}-\mathrm{Sb}}\right)$, effective charges $q_{\text {eff }}$ and corresponding atomic volumes $v_{\text {at }}$, and diffusion constants $D$. Standard deviations in parentheses.

\begin{tabular}{lccccc}
\hline \hline & $n_{\mathrm{X}}$ & $r_{\mathrm{X}-\mathrm{Sb}}(\AA)$ & $q_{\mathrm{eff}}(e)$ & $v_{\text {at }}\left(\AA^{3}\right)$ & $D\left(1 \times 10^{-5} \mathrm{~cm} / \mathrm{s}^{2}\right)$ \\
\hline $\mathrm{Ag}$ & 5.5 & 2.88 & $-0.35(0.18)$ & $27.5(1.8)$ & $7.330(0.006)$ \\
$\mathrm{In}$ & 5.4 & 3.15 & $+0.09(0.23)$ & $32.3(2.3)$ & $4.295(0.004)$ \\
$\mathrm{Sb}$ & 4.3 & 3.00 & $+0.04(0.08)$ & $30.7(2.1)$ & $4.122(0.001)$ \\
$\mathrm{Te}$ & 3.6 & 3.02 & $+0.11(0.03)$ & $32.8(2.2)$ & $4.806(0.002)$ \\
\hline \hline
\end{tabular}




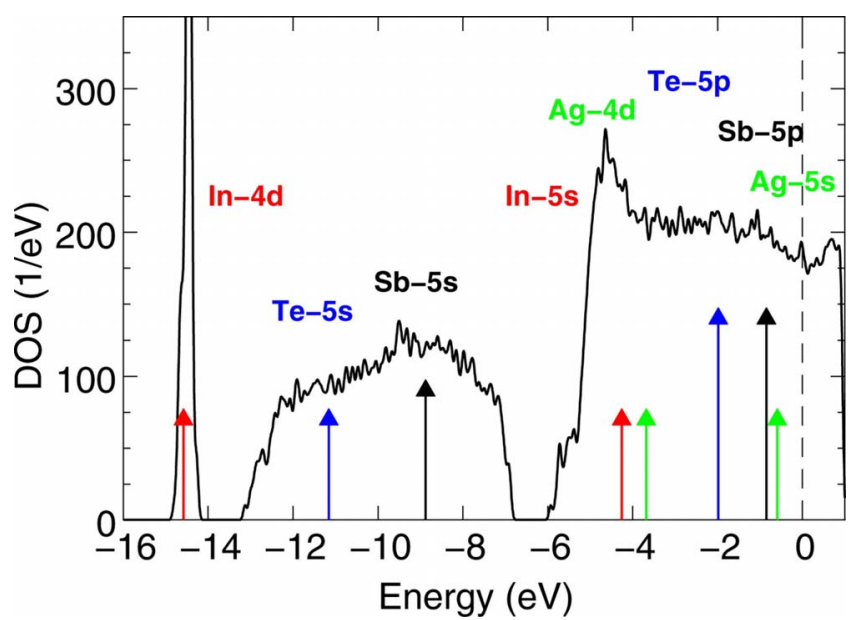

FIG. 4. (Color online) Electronic density of states of ( $\ell$-AIST) at $850 \mathrm{~K}$. Atomic eigenvalues are shifted (by $+4.0 \mathrm{eV}$ ) to align the In $4 d$ orbital with the peak at $-14.5 \mathrm{eV}$. Vertical dashed line: Fermi energy.

are commonly underestimated by DF calculations. The charges on the ions (Table I) are close to those of the constituent atoms: only the $\mathrm{Ag}$ anion differs significantly, and In is an electron donor.

The time-dependence of the mean square displacements of the elements ${ }^{19}$ leads to the diffusion constants $D$ in Table I. The enhanced mobility of $\mathrm{Ag}$ atoms is also evident in the angular distributions, ${ }^{19}$ where Ag has a wide variety of configurations. The vibrational density of states of the liquid shows little structure, apart from a broad peak $\sim 100 \mathrm{~cm}^{-1}$ arising from $\mathrm{Sb}$ vibrations.

AgInSbTe alloys are widely used in CD-RW and DVD-RW optical storage, and we have described extensive DF simulations (640 atoms in the unit cell, $\sim 50 \mathrm{ps,}$ $850 \mathrm{~K}$, and no adjustable parameters) on liquid $\mathrm{Ag}_{3.5} \mathrm{In}_{3.8} \mathrm{Sb}_{75.0} \mathrm{Te}_{17.7}$. The structure factor and PDF agree well with HEXRD measurements of the liquid at $589^{\circ} \mathrm{C}$ $(862 \mathrm{~K})$. The simulations provide the following information: (a) medium-range order is evident, (b) $\mathrm{Ag}$ and In atoms prefer to be near Te atoms rather than $\mathrm{Sb}$, (c) Ag atoms are the most mobile, have the shortest bonds and highest coordination number, and are anionic due to charge transfer to the Ag $5 s$ shell, (d) indium is slightly cationic (depletion from In $5 p$ ), and bonds with $\mathrm{Sb}$ and Te are longer and more flexible than those of Ag. These observations are presumably related to the phase change properties of AIST materials, and the combination of HEXRD and extensive DF simulations will surely provide insight into other complicated alloy systems.
The calculations were performed on IBM BlueGene/P and p6 575 computers in the Forschungszentrum Jülich with grants from the FZ Jülich and the John von Neumann Institute for Computing. We are grateful to T. Usuki and S. Kohara for helpful discussions and providing unpublished HEXRD data, and to M. Takata, T. Matsunaga, and N. Yamada for support.

${ }^{1}$ M. Wuttig and N. Yamada, Nature Mater. 6, 824 (2007).

${ }^{2}$ N. Yamada, E. Ohno, K. Nishiuchi, N. Akahira, K. Nagata, and M. Takao, J. Appl. Phys. 69, 2849 (1991).

${ }^{3}$ H. Iwasaki, Y. Ide, M. Harigaya, Y. Kageyama, and I. Fukimura, Jpn. J. Appl. Phys., Part 1 31, 461 (1992).

${ }^{4}$ S. Kohara, K. Kato, S. Kimura, H. Tanaka, T. Usuki, K. Suzuya, H. Tanaka, Y. Moritomo, T. Matsunaga, N. Yamada, Y. Tanaka, H. Suematsu, and M. Takata, Appl. Phys. Lett. 89, 201910 (2006).

${ }^{5}$ J. Akola and R. O. Jones, Phys. Rev. B 76, 235201 (2007); J. Phys.: Condens. Matter 20, 465103 (2008).

${ }^{6}$ S. Caravati, M. Bernasconi, T. D. Kühne, M. Krack, and M. Parrinello, Appl. Phys. Lett. 91, 171906 (2007).

${ }^{7}$ J. Hegedüs and S. R. Elliott, Nature Mater. 7, 399 (2008).

${ }^{8}$ Y. Fukuyama, N. Yasuda, J. Kim, H. Murayama, Y. Tanaka, S. Kimura, K. Kato, S. Kohara, Y. Moritomo, T. Matsunaga, R. Kojima, N. Yamada, H. Tanaka, T. Ohshima, and M. Takata, Appl. Phys. Express 1, 045001 (2008) and references therein.

${ }^{9}$ See, for example, H. J. Borg, P. W. M. Blom, B. A. J. Jacobs, B. Tieke, A. E. Wilson, I. P. D. Ubbens, and G. F. Zhou, Proc. SPIE 3864, 191 (1999).

${ }^{10}$ H. Tashiro, M. Harigaya, Y. Kageyama, K. Ito, M. Shinotsuka, K. Tani, A. Watada, N. Yiwata, Y. Nakata, and S. Emura, Jpn. J. Appl. Phys., Part 1 41, 3758 (2002).

${ }^{11}$ M. L. Lee, L. P. Shi, Y. Tian, C. L. Gan, and X. S. Miao, Phys. Status Solidi A 205, 340 (2008).

${ }^{12}$ C. Steimer, V. Coulet, W. Wełnic, H. Dieker, R. Detemple, C. Bichara, B. Beuneu, J. P. Gaspard, and M. Wuttig, Adv. Mater. (Weinheim, Ger.) 20, 1 (2008).

${ }^{13}$ T. Matsunaga, Y. Umetami, and N. Yamada, Phys. Rev. B 64, 184116 (2001).

${ }^{14} \mathrm{~T}$. Usuki, personal communication.

${ }^{15}$ Low Energy Neutrons and their Interaction with Nuclei and Matter, Landolt-Börnstein, New Series Vol. 16, edited by H. Schopper (Springer, Berlin, 2000), Chap. 6.

${ }^{16}$ CPMD V3.12 Copyright IBM Corp. 1990-2008, Copyright MPI für Festkörperforschung Stuttgart 1997-2001, http://www.cpmd.org

${ }^{17}$ J. P. Perdew, A. Ruzsinszky, G. I. Csonka, O. A. Vydrov, G. E. Scuseria, L. A. Constantin, X. Zhou, and K. Burke, Phys. Rev. Lett. 100, 136406 (2008).

${ }^{18}$ The density of $\mathrm{Sb}_{80} \mathrm{Te}_{20}$ [K. J. Singh, R. Satoh, and Y. Tsuchiya, J. Phys. Soc. Jpn. 72, 2546 (2003)] was adjusted for the sizes of Ag and In, and the energetics checked with short MD sequences at $850 \mathrm{~K}$ at different volumes. This density is close to the measured value of $\mathrm{Ag}_{3.4} \mathrm{In}_{3.7} \mathrm{Sb}_{76.4} \mathrm{Te}_{16.4}$ at $830 \mathrm{~K}$ [T. Matsunaga and N. Yamada, Jpn. J. Appl. Phys., Part 143 , 4704 (2004)].

${ }^{19}$ See EPAPS Document No. http://dx.doi.org/10.1063/1.3157166 for supplementary materials on the simulation, bond length and angle distributions, partial coordination numbers, cavities and atomic charges, and diffusion constants. 